\title{
Antihypertensive effect of a Chinese herbal formula (Salvia miltiorrhiza compound recipe) in hypertensive rats induced by high-fructose, high-salt, and high-fat diet
}

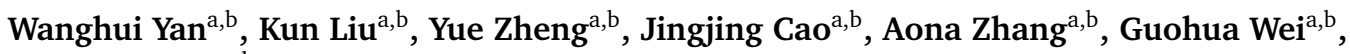 \\ Qingmei Zeng ${ }^{\mathrm{a}, \mathrm{b}, *}$ \\ a School of Food Science and Engineering, Hefei University of Technology, Hefei 230009, Anhui, China \\ b Engineering Research Centre of Bio-Process, Ministry of Education, Hefei University of Technology, \\ Hefei 230009, Anhui, China
}

*Corresponding author, e-mail: zengqingmei-1@163.com

Received 7 May 2018

Accepted 20 Jun 2018

\begin{abstract}
This study investigates the antihypertensive effect of a Chinese herbal formula (Salvia miltiorrhiza compound recipe, SMCR) and analysed its underlying mechanisms of action using hypertensive male Sprague Dawley rats. Hypertension was induced by the addition of $5 \%$ fructose to drinking water and placing the rats on a high-fat and high-salt diet. The animals were divided into six groups of eight rats each: the untreated control group, a high hypertensive group (HMG), SMCR three-dose groups (LDG, MDG, HDG), and a captopril positive control group. Body weight, systolic blood pressure (SBP), diastolic blood pressure (DBP), and heart rate were measured once a fortnight. At the end of the study, a number of indicators related to hypertension were detected. Kidney tissue was stained using H\&E. The hypertension model was successfully established by the high-fructose, high-salt and high-fat diet. Compared with the model group, SMCR could reduce SBP and DBP, and decrease the levels of renin, AngII, ET-1, TG, total cholesterol, LDL-C, left ventricular hypertrophy index, HW/BW, uric acid, and insulin $(p<0.05)$. SMCR could increase the levels of nitric oxide, eNOS and HDL-C $(p<0.05)$. There was no effect on weight or heart rate $(p<0.05)$, and there were no pathological changes in the kidney. The analysis of the experimental data shows that the SMCR has a significant effect on reducing hypertension, regulating abnormal lipid metabolism, and slightly affects insulin and uric acid levels.
\end{abstract}

KEYWORDS: diet-induced hypertensive rat model

\section{INTRODUCTION}

Hypertension is a disease condition characterized by increased arterial blood pressure, the pathogenesis of hypertension includes multiple organs involving the cardiorenal system. It is considered one of the main cardiovascular risk factors, being one of the most frequent reasons for medical consultation, both in primary care and in hospital emergency departments ${ }^{1}$. There are about 250 million hypertensive patients in China. The rising prevalence rate is correlated with a great increase in fast food consumption with high diet content of fat and salt and a dramatic increase of fructose intake in the last 100 years $^{2}$. High salt stimulation damaged the activity of $\mathrm{Na}^{+}-\mathrm{K}^{+}$pump in vascular smooth muscle cells, which led to the increase of $\mathrm{Na}^{+}$concentration in vascular smooth muscle cells and the activation of $\mathrm{Na}^{+}-\mathrm{Ca}^{+}$exchanger, resulting in an increase of $\mathrm{Ca}^{+}$ concentration in vascular smooth muscle cells and the depolarization of cell membrane. Eventually, vascular smooth muscle cells contract and blood pressure rises ${ }^{3,4}$. In addition, high-salt diet can also cause oxidative stress ${ }^{5}$, vascular endothelial dysfunction, and inflammatory response ${ }^{6}$. High fat diet cannot only cause fat deposition and obesity, but also increase the level of saturated fatty acids in the human body, thus making the triglyceride and cholesterol contents increase, which is related to the development of hypertension. Unlike other sugars, fructose rapidly causes adenosine triphosphate depletion and results in nucleotide turnover with generation of uric acid $^{7}$. Evidence from animal models supports the pathogenetic role of hyperuricaemia in the progression of hypertension and renal disease and its role as a causal risk factor for cardiovascular disease $^{8}$. High fructose diets also affect triglyceride levels ${ }^{9,10}$, lead to insulin sensitivity, and induce insulin resistance ${ }^{11,12}$; it is a harmful factor for the occurrence and development of hypertension. 
In China, Traditional Chinese Medicines have been used to treat cardiovascular diseases, especially hypertension. Abundant studies had been done on single herbal medicine, but many traditional Chinese medicine formulae were generally used according to experience, without scientific experimental evidence, so it is not clear whether there is a therapeutic effect and how they work. Thus this article will study a traditional Chinese medicine compound which called Salvia miltiorrhiza compound recipe (SMCR). This formula is a traditional Chinese medicine prescription for treating hypertension. It contains dried mature seeds of Cassia tora, Leguminosae (semen cassiae torae), Crataegus, Rosaceae (hawthorn), dried roots of S. miltiorrhiza Bge., Lamiaceae (S. miltiorrhiza), dried roots and rhizomes of Panax notoginseng (Burk.) F.H.Chen, Araliaceae (pseudo-ginseng), and dried rhizomes of Ligusticum chuanxiong Hort., Umbelliferae (L. chuanxiong) at a ratio of 1:1:3:2:1 (w/w). Hence the aim of this study is to build a successful hypertensive rat model, induced by high-fructose, high-salt and high-fat diet, and to study the antihypertensive effect and potential mechanism of SMCR.

\section{MATERIALS AND METHODS}

\section{Preparation of the plant extract}

Semen cassiae torae (ST), hawthorn (HR), S. miltiorrhiza (SM), pseudo-ginseng (PG), and L. chuanxiong (LC) were purchased from a local drugstore, namely, Hefei Big Pharmacy (Hefei, China). SMCR was prepared by mixing ST, HR, SM, PG, and LC at 1:1:3:2:1 wt ratio. Herbs were ground into powder and soaked with 4-fold volume of boiling water at $100^{\circ} \mathrm{C}$ for $1 \mathrm{~h}$. The process was repeated twice. The aqueous extract was filtered, pooled and further concentrated by rotary evaporation at $60^{\circ} \mathrm{C}$ under reduced pressure. Concentrated extract was lyophilized into powder and stored in desiccators at room temperature.

\section{Chemicals and reagents}

Captopril were purchased from a local drugstore, namely, Hefei Big Pharmacy (Hefei, China). Assay kits for determination of biochemical parameters, including nitric oxide (NO), uric acid (UA), total cholesterol (TC), triglyceride (TG), low-density lipoprotein cholesterol (LDL-C), high-density lipoprotein cholesterol (HDL-C), renin, Angiotensin II (AngII), endothelins-1 (ET-1), insulin, and nitric oxide synthase (eNOS) were purchased from Nanjing Jiancheng Biotechnology Institute (Nan- jing, China). All other reagents used throughout this study were of analytical grade and were obtained from Sinopharm Chemical Reagent (Shanghai, China).

\section{Experimental animals}

Male Sprague Dawley rats (5-week old, body weight 140-160 g) were purchased from the Experimental Animal Centre of Anhui Medical University (Hefei, China), animal license is No. SCXK (Anhui, China) 2017-001. During the experiments, the rats were housed in polypropylene cages under controlled environmental conditions (temperature of $23 \pm 2{ }^{\circ} \mathrm{C}$ and relative humidity of 40-60\%) and a $12 \mathrm{~h} / 12 \mathrm{~h}$ light/dark cycle. Before starting the experiment, the rats were acclimatized for one week. During the whole experiment, all the animals were treated according to the guidelines of the National Institute of Health, which is targeted at the care and use of laboratory animals, and their experimental use was approved by the Animal Ethics Committee of Hefei University of Technology.

\section{Experimental design}

Rats were allowed access to water ad libitum and a standard laboratory diet (60\% cereals, $33 \%$ protein, $3 \%$ grease, $4 \%$ amino acids). After acclimation for 1 week, the rats were divided into two groups: control group (CG, $n=8$ ), hypertensive group (HG, $n=40$ ). Throughout the experiment, rats in the CG were fed the standard laboratory chow diet for 14 weeks, while rats in the HG ( $n=40$ ) were given $10 \%$ of fructose water and fed a high-salt and high-fat diet $63 \%$ standard laboratory diet with $20 \%$ lard added, $12 \%$ egg, $5 \%$ salt, prepared in our laboratories) for 8 weeks and then randomly divided into five groups $(n=8$ each): hypertension model group (HMG), SMCRtreated hypertension groups which including SMCRlow-dose group (LDG), SMCR-middle-dose group (MDG), and SMCR-high-dose group (HDG), and captopril positive control group (CPCG). The five groups were still fed a high-fructose, high-salt, and high-fat diet for 6 weeks.

Starting from the ninth week, CG and HMG were treated by gastric gavage with distilled water at dose of $100 \mathrm{mg} / \mathrm{kg}$. LDG, MDG, and HDG were treated by gastric gavage with SMCR at doses of 1.5 , 3 , and $6 \mathrm{~g} \mathrm{~kg}^{-1} \mathrm{~d}^{-1}$. CPCG was treated by gastric gavage with captopril at dose of $7.5 \mathrm{mg} \mathrm{kg}^{-1} \mathrm{~d}^{-1}$. Samples were treated by gastric gavage into rats continuously once per day (at 9:00 am) for 6 weeks. 

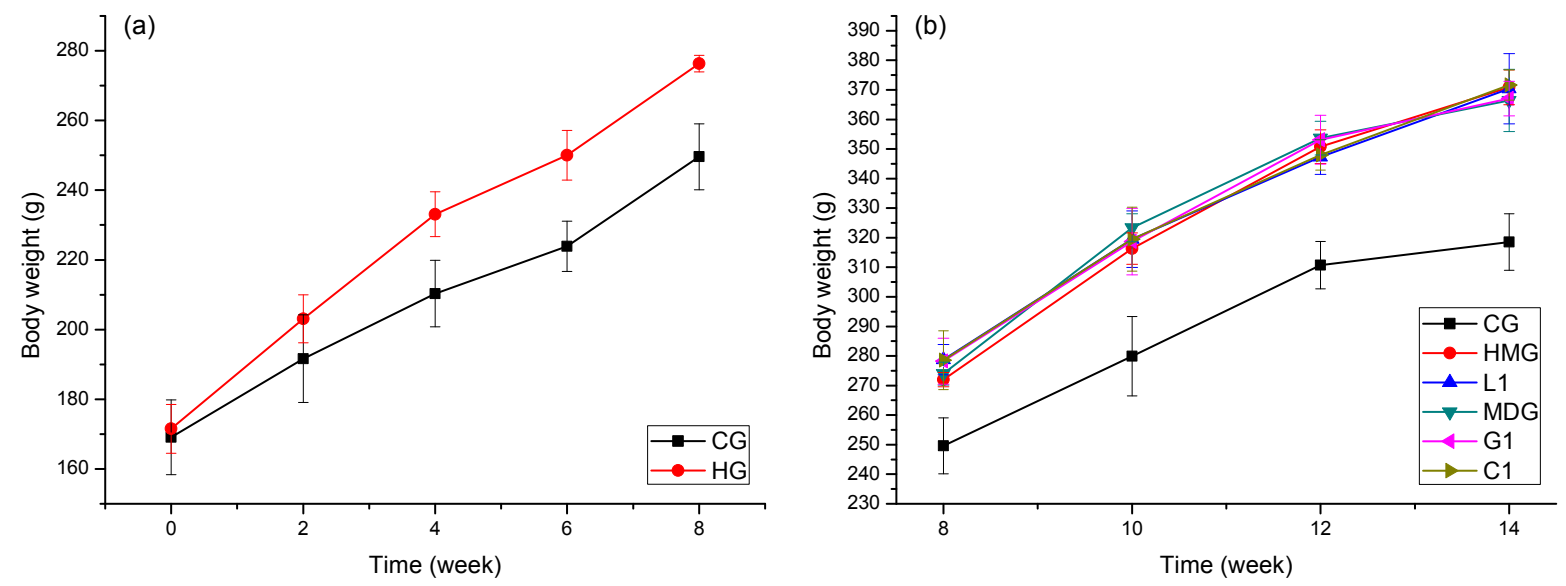

Fig. 1 (a) Effect of high-fructose, high-fat, and high-salt diet on the body weight of hypertensive rats from 0 weeks to 8 weeks. (b) Effect of SMCR on the body weight of hypertensive rats from 8 weeks to 14 weeks. In this figure and the following, data are shown as mean $\pm \operatorname{SD}(n=8)$.

Systolic blood pressure (SBP), diastolic blood pressure (DBP), and heart rate (HR) were measured by tail-cuff method using the ZH-HX-Z series rat tail blood pressure system (Anhui Zhenghua Biological instrument equipment Co., Huaibei, China), once a fortnight, on the $0,2,4,6,8,10,12$, and 14 weeks during the experiments. Body weight was also measured once a fortnight.

\section{Determination of biochemical parameters}

At the end of the six weeks, the rats were anaesthetized via urethane ( $20 \%$ concentration, $1.5 \mathrm{~g} / \mathrm{kg}$ ) intraperitoneal injection. The anaesthetized rats were fixed on the operating table, abdominal aorta was exposed to collect blood, meanwhile, the kidney and heart were quickly removed, washed, and blotted for further use. Part of the obtained blood was placed at $4^{\circ} \mathrm{C}$ for about $3 \mathrm{~h}$. After blood clotting and centrifugation $\left(1000 \mathrm{~g}, 4^{\circ} \mathrm{C}, 15 \mathrm{~min}\right)$, serum was used for NO, TG, TC, LDL-C, HDL-C, insulin, and UA detection using commercially available kits. The rest of the blood was collected in EDTA anticoagulant tube. After centrifugation $\left(1000 \mathrm{~g}, 4^{\circ} \mathrm{C}\right.$, $15 \mathrm{~min}$ ), plasma was used for ET-1, AngII, renin, and eNOS detection using the commercially available kits. The whole heart was weighed, and the atrium, the large vessels, and pericardial tissues were removed. The left ventricle including ventricular septum was separated along the interventricular septum, cleaned with filter paper, drained, and moisture was added. The left ventricular portion including the ventricular septum weight was measured and the heart coefficient and left ventricular hypertrophy index were calculated. Fresh kidney tissue was collected and immersed in $4 \%$ formaldehyde for $48 \mathrm{~h}$ and then removed and dehydrated before embedding. The paraffin-embedded tissues were cut into $5-\mu \mathrm{m}$ sections, stained with haematoxylin and eosin (HE) according to standard techniques and observed under an optical microscope at 100x for detecting the degree of pathological changes and recovery.

\section{Statistical analysis}

The experimental data were expressed as a mean \pm standard error. All the statistical analyses including one-way ANOVA and Duncan's test were processed using IBM SPSS STATISTICS 22.0. The differences were considered significant at $p<0.05$ and $p<0.01$.

\section{RESULTS}

\section{Effect of SMCR on the body weight of hypertensive rats}

Changes in the body weight of rats were investigated once a fortnight. As shown in Fig. 1, the body weight of rats in all the groups had increased with increasing days throughout the experiment. However, high-fructose, high-fat, and high-salt diet feeding led to a significant increase in body weight compared with those in CG group since the fourth week ( $p<0.01$, Fig. 1a). After 6 weeks of treatment, SMCR and captopril administration cannot slow down body weight gain, and there was no significant difference compared with the HMG group ( $p<0.05$, Fig. 1b) Thus the body weight values of the treatment groups were significantly higher than that of the CG ( $p<0.01$, Fig. 1b). 

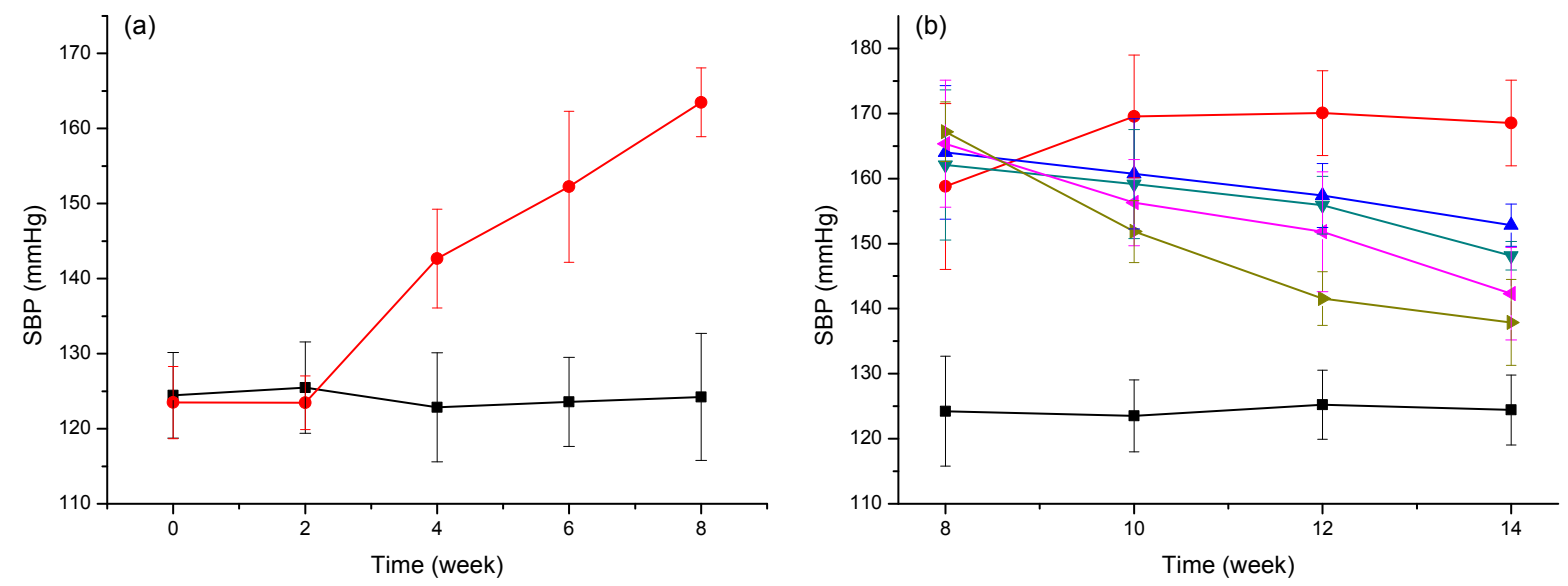

Fig. 2 (a) Effect of high-fructose, high-fat, and high-salt diet on the systolic blood pressure of hypertensive rats from 0 weeks to 8 weeks. (b) Effect of SMCR on the systolic blood pressure of hypertensive rats from 8 weeks to 14 weeks. Legend as in Fig. 1.
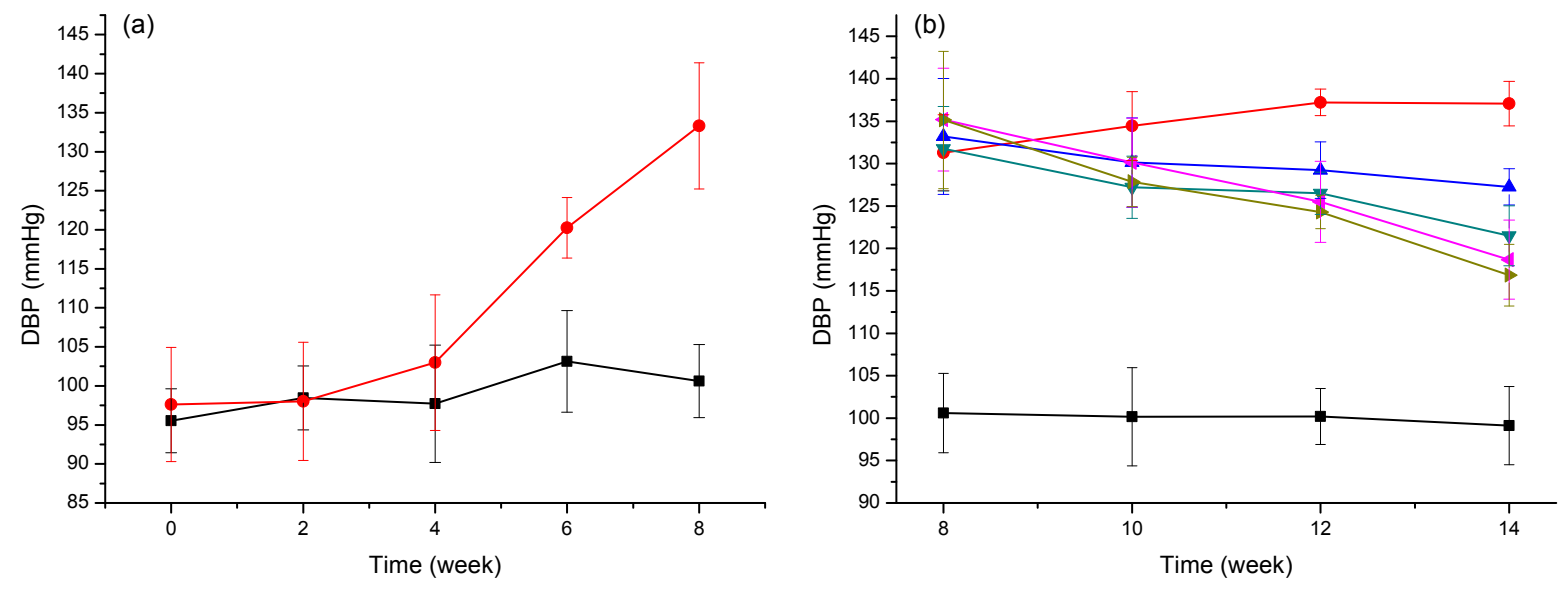

Fig. 3 (a) Effect of high-fructose, high-fat, and high-salt diet on the diastolic blood pressure of hypertensive rats from 0 weeks to 8 weeks. (b) Effect of SMCR on the diastolic blood pressure of hypertensive rats from 8 weeks to 14 weeks. Legend as in Fig. 1.

\section{Effect of SMCR on systolic blood pressure of hypertensive rats}

Changes of systolic blood pressure (SBP) in rats before and after intragastric administration are shown in Fig. 2, and the SBP of rats were investigated once a fortnight. The SBP of the CG remained nearly unchanged during the entire experimental period. The HG had a significant increase in SBP compared with the CG because of the high-fructose, high-salt, and high-fat diet feeding ( $p<0.01$, Fig. 2a). From the ninth week, gastric administration began, and after 6 weeks of gastric administration, though the SBP of the treatment group was significantly higher than that of the CG ( $p<0.01$, Fig. $2 b)$. It was also significantly lower than that of the HMG ( $p<0.01$, Fig. 2b) and the antihypertensive effect of CPCG is the best and fastest.

\section{Effect of SMCR on diastolic blood pressure of hypertensive rats}

Changes of diastolic blood pressure (DBP) in rats before and after gastric administration are shown in Fig. 3. The DBP of rats were investigated once a fortnight. The DBP of the CG showed only slight fluctuation during the entire experimental period. The HG had a significant increase in DBP compared with the CG because of the high-fructose, high-salt, and high-fat diet feeding ( $p<0.01$, Fig. 3a). From the ninth week, gastric administration began, and after 6 weeks of gastric administration, though the DBP of the treatment group was significantly higher than that of the CG ( $p<0.01$, Fig. 3b), it was also 

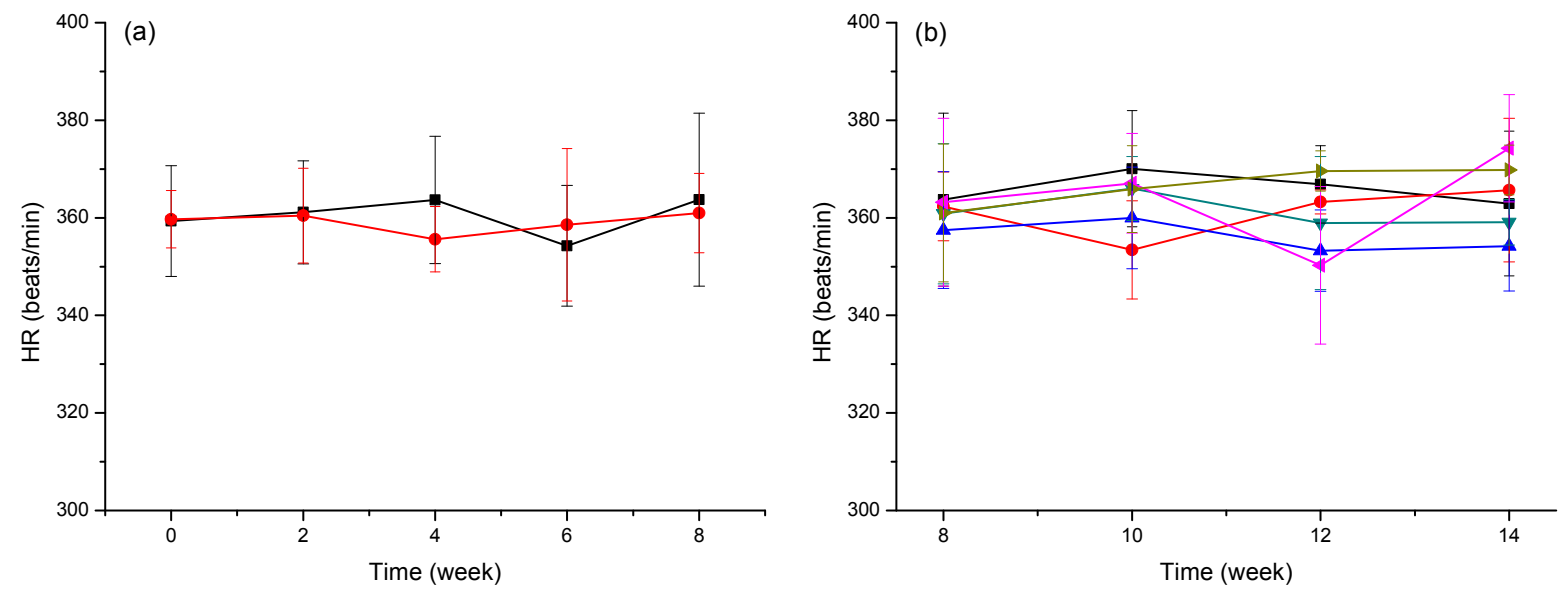

Fig. 4 (a) Effect of high-fructose, high-fat, and high-salt diet on the heart rate of hypertensive rats from 0 weeks to 8 weeks. (b) Effect of SMCR on the heart rate of hypertensive rats from 8 weeks to 14 weeks. Legend as in Fig. 1.

Table 1 Effect of SMCR on TC, TG, LDL-C, HDL-C, LVHI and HW/BW of hypertensive rats $(n=8)$.

\begin{tabular}{lcccccc}
\hline Group & TG (mmol/l) & TC (mmol/l) & LDL-C (mmol/l) & HDL-C (mmol/l) & LVHI $(\mathrm{mg} / \mathrm{g})$ & HW/BW (\%) \\
\hline CG & $0.61 \pm 0.09$ & $1.36 \pm 0.08$ & $0.58 \pm 0.11$ & $1.18 \pm 0.08$ & $1.96 \pm 0.05$ & $0.29 \pm 0.01$ \\
HMG & $2.98 \pm 0.12^{* * *}$ & $2.16 \pm 0.15^{* *}$ & $1.29 \pm 0.11^{* *}$ & $0.82 \pm 0.04^{* * *}$ & $2.65 \pm 0.20^{* *}$ & $0.35 \pm 0.02^{* * *}$ \\
LDG & $2.43 \pm 0.09^{* * \# \#}$ & $1.90 \pm 0.09^{* * \# \#}$ & $1.06 \pm 0.08^{* * \# \#}$ & $0.89 \pm 0.04^{* *}$ & $2.59 \pm 0.22^{* *}$ & $0.33 \pm 0.02^{* * \# \#}$ \\
MDG & $2.09 \pm 0.10^{* * \# \#}$ & $1.76 \pm 0.07^{* * \# \#}$ & $0.93 \pm 0.07^{* * \# \#}$ & $0.97 \pm 0.04^{* * \# \#}$ & $2.14 \pm 0.12^{\# \#}$ & $0.28 \pm 0.05^{\# \#}$ \\
HDG & $1.39 \pm 0.12^{* * \# \#}$ & $1.53 \pm 0.08^{* \# \#}$ & $0.69 \pm 0.05^{* \# \#}$ & $1.09 \pm 0.10^{* \# \#}$ & $2.04 \pm 0.17^{\# \#}$ & $0.29 \pm 0.02^{\# \#}$ \\
CPCG & $2.94 \pm 0.13^{* * *}$ & $2.08 \pm 0.07^{* *}$ & $1.27 \pm 0.08^{* *}$ & $0.85 \pm 0.07^{* *}$ & $2.54 \pm 0.04^{* *}$ & $0.34 \pm 0.05^{* *}$ \\
\hline
\end{tabular}

" $p<0.05$ and ${ }^{* *} p<0.01$ as compared with CG; ${ }^{\#} p<0.05$ and ${ }^{\# \#} p<0.01$ as compared with HMG.

Data are mean \pm SD $(n=8)$.

significantly lower than that of the HMG ( $p<0.01$, Fig. 3b). The antihypertensive effect of CPCG was similar to that of HDG.

\section{Effect of SMCR on heart rate of hypertensive rats}

Changes of heart rate (HR) in rats before and after intragastric administration are shown in Fig. 4. The $\mathrm{HR}$ of rats were investigated once a fortnight. As shown in Fig. 4, The HR of the rats showed only slight fluctuation during the entire experimental period. Before and after gastric administration, there was no significant different in the HR of rats in the treated groups compared with those in CG ( $p>0.05$, Fig. 4a, 4b). This indicated that SMCR have no significant effect on the HR of rats.

\section{Effect of SMCR on TC, TG, LDL-C, HDL-C, LVHI and $\mathrm{HW} / \mathrm{BW}$ of hypertensive rats}

In Table 1 , it was found that the TG, TC, LDL-C, left ventricular hypertrophy index (LVHI) and heart weight/body weight (HW/BW) of the HMG was significantly higher than that of CG $(p<0.01)$, and the
HDL-C was significantly lower than that of CG ( $p<$ 0.01). This suggests that high fructose, high-salt and high-fat diet caused lipid metabolism disorder and myocardial abnormality. After administration of drugs, the TC, TG, LDL-C, LVHI, and HW/BW in SMCR groups were significantly lower than that of HMG $(p<0.01)$. Meanwhile, the HDL-C in MDG and HDG were significantly higher than that of HMG $(p<0.01)$. The CPCG has little effect on TG, TC, LDL-C, HDL-C, LVHI, and HW/BW $(p<0.05)$.

\section{Effect of SMCR on renin, AngII and ET-1 in hypertensive rats}

In Fig. 5, the plasma renin level in the HMG was significantly higher than that in the CG $(p<0.01)$. Although there was a significant difference between the CG and the SMCR treatment groups $(p<0.01)$, the plasma renin level in the SMCR treatment groups were significantly lower than that in the HMG $(p<0.01)$. The effect of the CPCG was the best. The declined ratios of renin levels in the SMCR treatment groups were in the range of $8 \%$ to $18 \%$. Comparing the renin levels with the different doses 


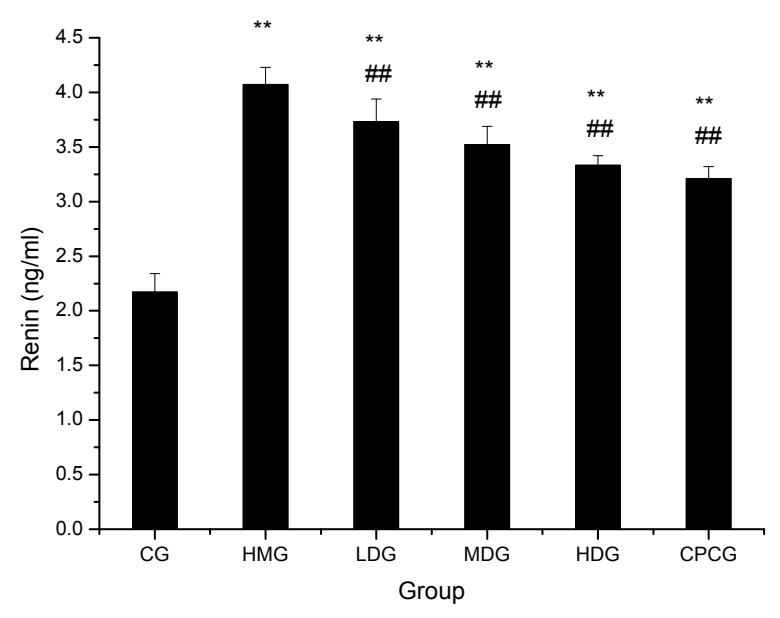

Fig. 5 Effect of SMCR on renin in hypertensive rats (in this figure and the following: ${ }^{*} p<0.05$ and ${ }^{* *} p<0.01$ as compared with CG, ${ }^{\#} p<0.05$ and ${ }^{\# \#} p<0.01$ as compared with HMG).

of the SMCR treatments, in the high-dose groups were significantly lower than those in the low-dose groups $(p<0.01)$. HDG declined by $11 \%$ compared with LDG.

In Fig. 6, the plasma AngII level in the HMG was significantly higher than that in the CG $(p<0.01)$, although there was a significant difference between the CG and the treatment groups, the plasma AngII level in the treatment groups were significantly lower than that in the HMG $(p<0.01)$. The effect of the CPCG was the best. The declined ratios of AngII levels in the treatment groups were in the range of $15 \%$ to $38 \%$. Comparing the AngII levels with the different doses of the SMCR treatments, in the highdose groups were significantly lower than those in

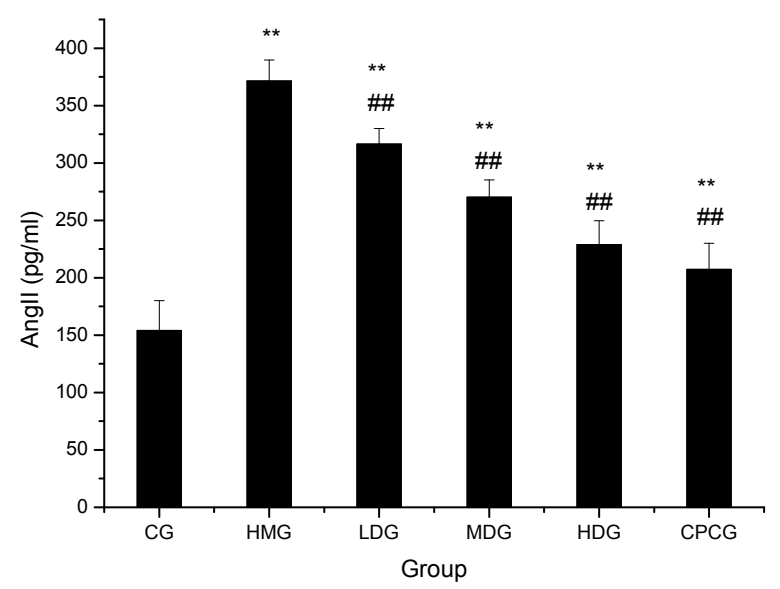

Fig. 6 Effect of SMCR on AngII in hypertensive rats.

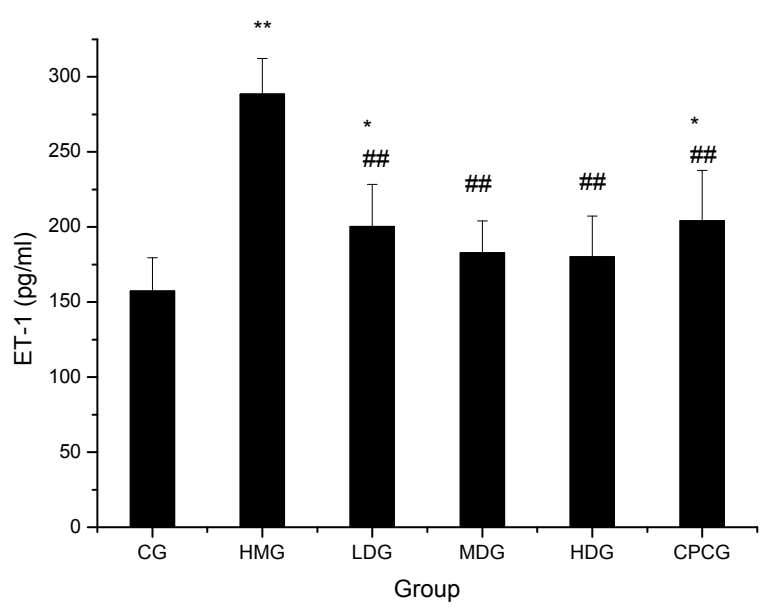

Fig. 7 Effect of SMCR on ET-1 in hypertensive rats.

the low-dose groups $(p<0.01)$. HDG declined by $28 \%$ compared with LDG. The effect of the HDG was similar to that of the CPCG, and there was no significant difference.

In Fig. 7, the plasma ET-1 level in the HMG was significantly higher than that in the CG ( $p<$ 0.01). After treatment, the plasma ET-1 level was significantly reduced compared with the HMG ( $p<$ 0.01). The declined ratios of ET-1 levels in the treatment groups were in the range of $31 \%$ to $38 \%$. Comparing the ET-1 levels with the different doses of the SMCR treatments, there was no significant difference between the HDG and LDG. The effect of the CPCG was similar to that of the LDG, although the level of ET-1 was decreased, it was significant compared with the CG $(p<0.05)$.

In conclusion, SMCR can reduce the level of renin, AngII, and ET-1 in the plasma of hypertensive rats induced by high-fructose, high-salt, and highfat.

\section{Effect of SMCR on NO and eNOS in hypertensive rats}

Fig. 8 shows that the serum nitric oxide (NO) level in the HMG was significantly lower than that in the CG $(p<0.05)$. After treatment, there was no significant difference between the treatment groups and the CG. The increased ratios of NO levels in the SMCR treatment groups were in the range of $18 \%$ to $52 \%$ compared with the HMG. The serum NO level in the HDG and CPCG were significantly higher than that in the HMG $(p<0.05)$.

In Fig. 9, the plasma eNOS level in the HMG was significantly lower than that in the CG $(p<$ 0.01 ), although there was a significant difference 


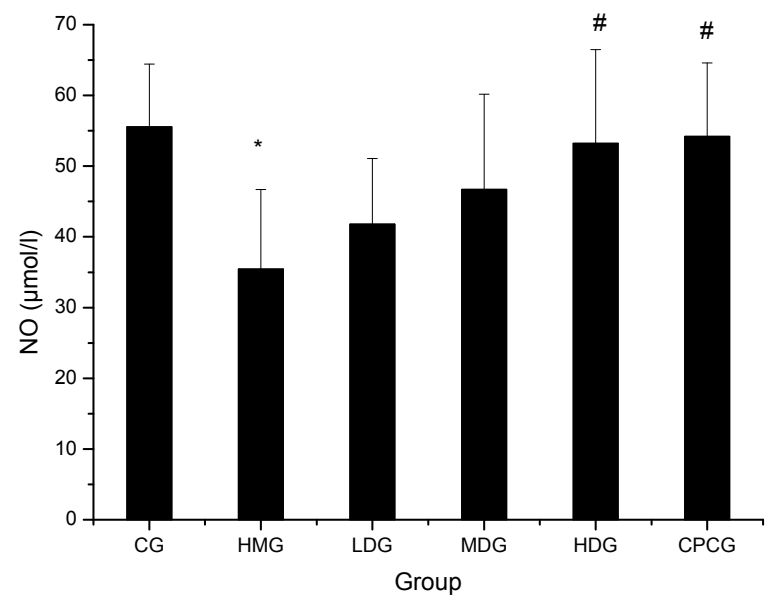

Fig. 8 Effect of SMCR on NO in hypertensive rats.

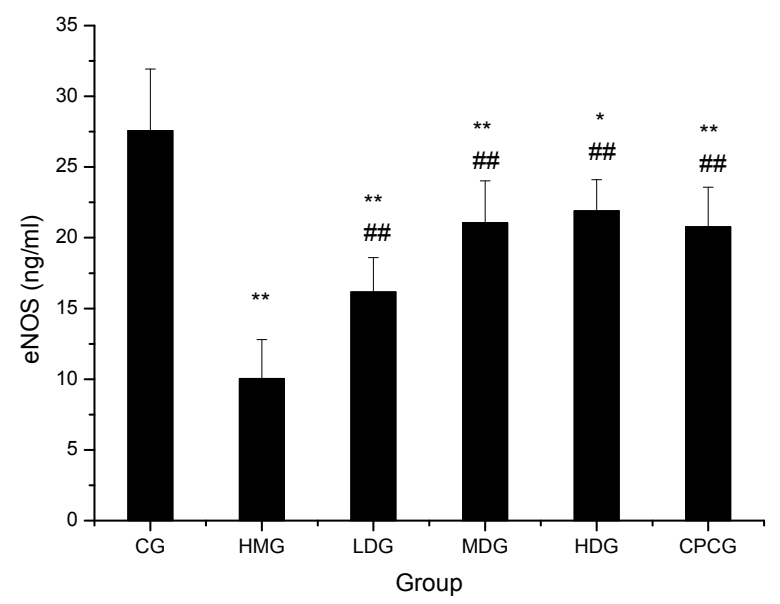

Fig. 9 Effect of SMCR on eNOS in hypertensive rats.

between the CG and the treatment groups, the plasma eNOS level in the treatment groups were significantly higher than that in the HMG $(p<$ 0.01 ). The increased ratios of eNOS levels in the treatment groups were in the range of $61 \%$ to $118 \%$. Comparing the eNOS levels with the different doses of the SMCR treatments, there was no significant difference between the HDG and MDG, but there was significant difference between the HDG and LDG, HDG increased by 35\% compared with LDG. The effect of the CPCG was similar to that of the MDG. The effect of the HDG was the best.

\section{Effect of SMCR on insulin and UA in} hypertensive rats

In Table 2, compared with the CG, the insulin level in all the treatment groups increased significantly $(p<0.01)$. After intragastric administration, the
Table 2 Effect of SMCR on insulin and UA of hypertensive rats $(n=8)$.

\begin{tabular}{lcc}
\hline Group & Insulin $(\mathrm{pg} / \mathrm{ml})$ & UA $(\mu \mathrm{mol} / \mathrm{l})$ \\
\hline CG & $322 \pm 12$ & $170 \pm 19$ \\
HMG & $373 \pm 13^{* *}$ & $208 \pm 31^{*}$ \\
LDG & $364.8 \pm 9.4^{* *}$ & $198 \pm 24$ \\
MDG & $356 \pm 11^{* * \#}$ & $189 \pm 22$ \\
HDG & $337.7 \pm 9.8^{* \# \#}$ & $187 \pm 25$ \\
CPCG & $365.2 \pm 8.1^{* *}$ & $185 \pm 28$ \\
\hline
\end{tabular}

" $p<0.05$ and ${ }^{* *} p<0.01$ as compared with CG.

${ }^{\#} p<0.05$ and ${ }^{\# \#} p<0.01$ as compared with HMG.

Data are shown as mean $\pm \operatorname{SD}(n=8)$.

MDG $(p<0.05)$ and HDG $(p<0.01)$ had a significant decrease compared with the HMG, meanwhile, there was no significant change in the LDG and CPCG compared with the HMG.

In addition, compared with the CG, the serum uric acid (UA) level in the HMG increased significantly $(p<0.05)$, but there was no significant difference between the treatment groups and the CG. Meanwhile, there was no significant difference between the treatment groups and the HMG.

\section{Histopathological observations in kidney tissue} sections

In Fig. 10, the results showed that high-fructose, high-salt, and high-fat diet had no significant effect on the kidney; the kidney showed no pathological changes and had only slight cell oedema. This might be that it was a long-term process of organ lesion. In this study, although the blood pressure rises quickly, the modelling process is short, so there were no lesions.

\section{DISCUSSION}

In the present study, we proved that the traditional Chinese medicine prescription of SMCR can significantly reduce SBP (Fig. 2) and DBP (Fig. 3) in hypertensive rats. The effect of the highest dose of lowering blood pressure was the same as that of captopril, a common antihypertensive drug. We also found that SCMR had no significant effect on body weight (Fig. 1) and heart rate (Fig. 4). To find out the possible mechanism of SMCR decreasing hypertension, the related factors in the blood were detected, and the heart and kidneys were also investigated.

First, we refer to the renin-angiotensin-aldosterone system, whose changes play a major role in the control of hypertension, and antagonists or inhibitors acting on the RAAS system are effective 

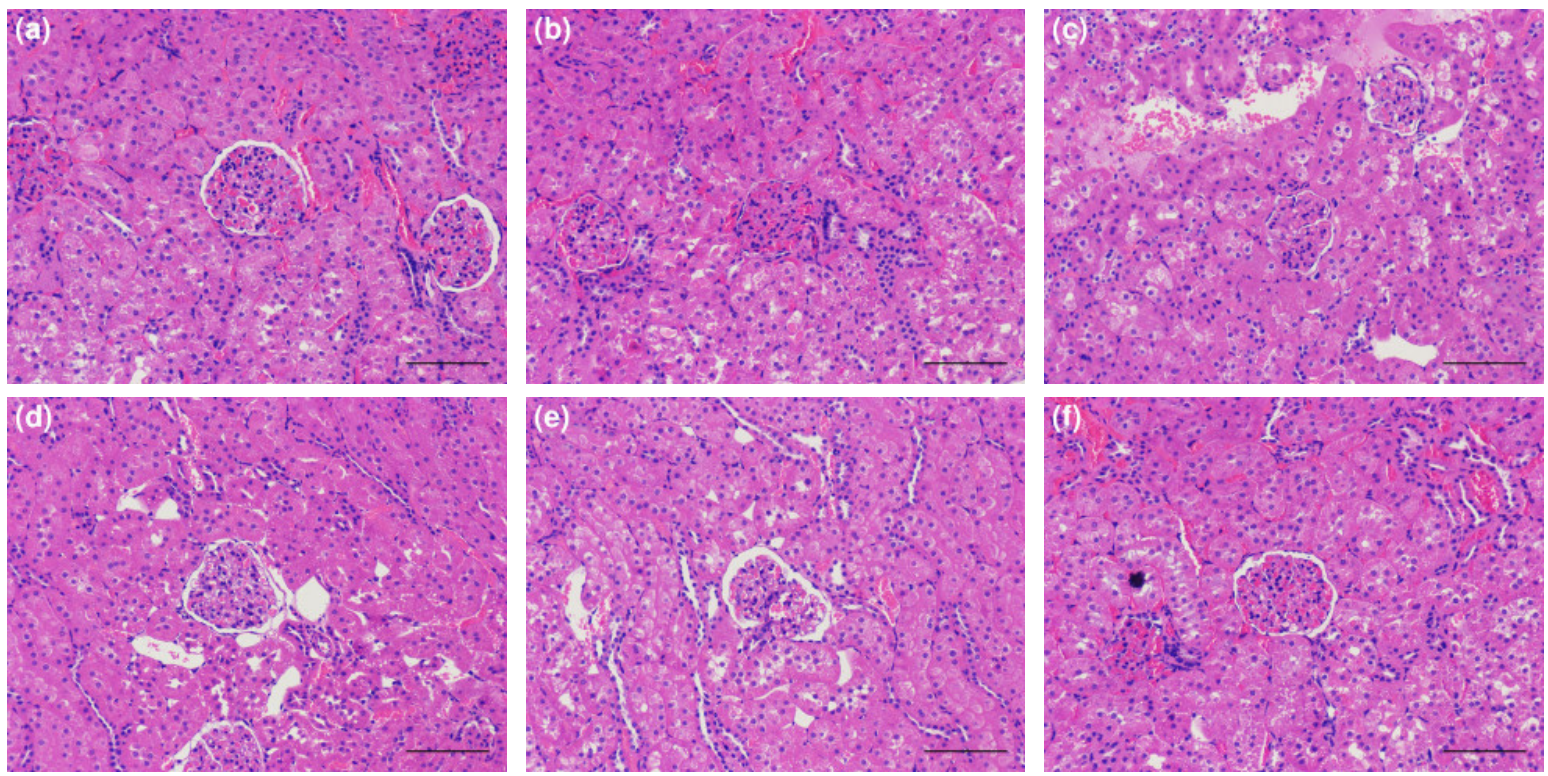

Fig. 10 Kidney tissue morphology of sections dyed with H\&E at 100×. (a) CG, the control group; (b) MG, the hypertensive model group; (c) LDG, the SMCR-low-dose group; (d) MDG, the SMCR-middle-dose group; (e) HDG, the SMCR-high-dose group; (f) CPCG, captopril positive control group.

in the treatment of hypertension ${ }^{13}$. Meanwhile, changes in the structure and function of blood vessels associated with hypertension ${ }^{14}$, which is also related to the over-activation of RAAS system ${ }^{15}$. In this study, SMCR decreased the levels of renin (Fig. 5) and AngII (Fig. 6) in the plasma, and significantly regulated the symptoms of left ventricular hypertrophy (Table 1). This is consistent with previous studies, which reported that $S$. miltiorrhiza and $L$. chuanxiong can regulate the RAS system and reduce the level of renin and AngII, mainly by regulating the mRNA expression of renin and AngII in myocardium and aorta ${ }^{16,17}$. There were reports which suggest that the regulation of $S$. miltiorrhiza on left ventricular hypertrophy in rats may be related to the adjustment and improvement of local catecholamine and oxygen free radical metabolism in the heart, and may also be related to the inhibition of metabolism of RAS in vivo ${ }^{18}$. Other studies have shown that $P$. notoginseng saponins can regulate the RAS system, increase left ventricular systolic and diastolic function, reduce peripheral resistance, and protect and improve cardiac function in rats ${ }^{19}$.

Secondly, the occurrence of hypertension is also related to a variety of active substances which is secreted by vascular endothelial cells. NO and ET1 are the two main endothelium-derived vasoactive factors, and they have opposite effects. NO can dilate blood vessels, regulate blood pressure, improve regional blood flow, inhibit platelet ag- gregation and inhibit smooth muscle cell proliferation. Endothelial nitric oxide synthase (eNOS) is the only rate-limiting enzyme for the production of NO. The endothelium-dependent vasodilation induced by endothelium-mediated vasodilation can disappear after given the inhibitor of eNOS. ET-1 is the strongest vasoconstrictor and pressure substance. Vascular endothelium regulates vascular function by regulating the balance of ET-1 and NO. In this study, SMCR decreased the level of ET-1 (Fig. 7), increased the levels of eNOS (Fig. 9) and NO (Fig. 8), which was consistent with the previous studies. S. miltiorrhiza extract can improve atherosclerotic thrombosis in vitro and in vivo through endothelial nitric oxide synthase and cyclooxygenase pathway ${ }^{20}$. Injection of compound S. miltiorrhiza can improve vascular endothelial function in patients with coronary heart disease. One of its mechanisms is to regulate the mRNA expression of endothelial nitric oxide synthase and ET- ${ }^{21}$. We therefore studied the effects of SMCR on blood lipids, insulin and UA. Dyslipidaemia is mainly related to highfat and high-sugar diets. Insulin and UA abnormalities are mainly associated with high fructose diets. Unlike other sugars, fructose can quickly lead to the consumption of adenosine triphosphate, leading to the conversion of nucleotides to uric acid. In this study, TG, TC, LDL-C (Table 1) and insulin (Table 2) decreased significantly, while HDLC (Table 1) increased after administration. Mean- 
while, there was no significant change in uric acid (Table 2). These may be related to the activity of Cassia seed and hawthorn. Semen cassiae may regulate hepatic lipid homoeostasis by enhancing the phosphorylation of AMP-activated protein kinase (AMPK) and its primary downstream targeting enzyme, acetyl-CoA carboxylase, up-regulating the gene expression of carnitine palmitoyl transferase1, and down-regulating sterol regulatory element binding protein 1 and fatty acid synthase protein levels ${ }^{22}$. Hawthorn leaf extract increased the gene expression of peroxisome proliferator activated receptor $\alpha$, down-regulated sterol regulatory element binding protein- $1 \mathrm{cr}$, and increased the gene expression of superoxide dismutase 1 , superoxide dismutase 2, and glutathione peroxidase $3^{23}$. S. miltiorrhiza have also been proved to have protective effects on insulin resistance, mainly by reducing oxidative stress ${ }^{24}$. Some research had proved that vasorelaxant action was produced primarily by inhibition of $\mathrm{Ca}^{2+}$ influx in the vascular smooth muscle cells and a small component was mediated by the opening of $\mathrm{K}^{+}$channels ${ }^{25}$.

In conclusion, our results showed that SMCR could significantly reduce blood pressure, improve left ventricular hypertrophy and regulate lipid and insulin abnormalities in hypertensive rats. SMCR is a compound drug with multi-target coordination effect. The results suggest that its possible mechanism of lowering blood pressure might be to regulate the mRNA expression of renin, AngII, ET-1 and eNOS in myocardium and aorta, inhibit lipids accumulation via the activation of AMPK, up regulate the expression of carnitine palmitic transferase1, down regulate the sterol regulator element binding protein 1 and fatty acid synthase, and inhibit the calcium influx in vascular smooth muscle cells. These findings may provide theoretical evidence for SMCR to be used scientifically.

Acknowledgements: This study was funded by the National Natural Science Foundation of China (Grant Nos. 31371844; 31071556) and Science and Technology Department of Anhui province, PR of China (Grant No. 1301032155).

\section{REFERENCES}

1. James PA, Oparil S, Carter BL, Cushman WC, Dennison-Himmelfarb C, Handler J, Lackland DT, et al (2014) 2014 Evidence-based guideline for the management of high blood pressure in adults: report from the panel members appointed to the Eighth Joint National Committee (JNC 8). JAMA 311, 507-20.
2. Pereira MA, Kartashov AI, Ebbeling CB, Van Horn L, Slattery ML, Jacobs DR Jr, Ludwig DS (2005) Fastfood habits, weight gain, and insulin resistance (the CARDIA study): 15-year prospective analysis. Lancet 365, 36-42.

3. Adrogue H, Madias N (2007) Sodium and potassium in the pathogenesis of hypertension. New Engl J Med 356, 1966-78.

4. Handelman G, Kotanko P, Levin N W (2007) Mechanisms of hypertension. New Engl J Med 357, 827-9.

5. Banday AA, Lau YS, Lokhandwala MF (2008) Oxidative stress causes renal dopamine D1 receptor dysfunction and salt-sensitive hypertension in SpragueDawley rats. Hypertension 51, 367-75.

6. Baszczuk A, Kopczyński Z, Thielemann A (2014) Endothelial dysfunction in patients with primary hypertension and hyperhomocysteinemia. Postępy Hig Med Dośw 68, 91-100 [in Polish].

7. Johnson RJ, Nakagawa T, Sanchez-Lozada LG, Shafiu M, Sundaram S, Le M, Ishimoto T, et al (2013) Sugar, uric acid, and the etiology of diabetes and obesity. Diabetes 62, 3307-15.

8. Filiopoulos V, Hadjiyannakos D, Vlassopoulos D (2012) New insights into uric acid effects on the progression and prognosis of chronic kidney disease. Ren Fail 34, 510-20.

9. Livesey G, Taylor R (2008) Fructose consumption and consequences for glycation, plasma triacylglycerol, and body weight: meta-analyses and metaregression models of intervention studies. Am J Clin Nutr 88, 1419-37.

10. Sievenpiper JL, Carleton AJ, Chatha S, Jiang HY, de Souza RJ, Beyene J, Kendall CW, Jenkins DJ (2009) Heterogeneous effects of fructose on blood lipids in individuals with type 2 diabetes: systematic review and meta-analysis of experimental trials in humans. Diabetes Care 32, 1930.

11. D'Angelo G, Elmarakby AA, Pollock DM, Stepp DW (2005) Fructose feeding increases insulin resistance but not blood pressure in Sprague-Dawley rats. Hypertension 46, 806-11.

12. Collins FS (1992) Cystic fibrosis: molecular biology and therapeutic implications. Science 256, 774-9.

13. Atlas SA (2007) The renin-angiotensin aldosterone system: pathophysiological role and pharmacologic inhibition. J Manag Care Spec Pharm 13(8 Supp B), 9-20.

14. Heagerty AM, Aalkjaer C, Bund SJ, Korsqaard N, Mulvany MJ (1993) Small artery structure in hypertension. Dual processes of remodeling and growth. Hypertension 21, 391-7.

15. Crowley SD, Gurley SB, Herrera MJ, Ruiz P, Griffiths R, Kumar AP, Kim HS, et al (2006) Angiotensin II causes hypertension and cardiac hypertrophy through its receptors in the kidney. Proc Natl Acad Sci USA 103, 17985-90.

16. Fu Y, Liu Q, Ma Z, Chen Y (2016) Effects of Gastrodia 
elata and Ligusticum chuanxiong on RAS in spontaneously hypertensive rats. Lishizhen Med Mater Med Res 27, 854-6 [in Chinese].

17. Kang DG, Yun YG, Ryoo JH, Lee HS (2002) Antihypertensive effect of water extract of danshen on renovascular hypertension through inhibition of the renin angiotensin system. Am J Chin Med 30, 87-93.

18. Han S, Zheng Z, Ren D (2002) Effects of Salvia miltiorrhiza on left ventricular hypertrophy and cardiac aldosterone in spontaneously hypertensive rats. J Huazhong Univ Sci Tech Med Sci 22, 302-4.

19. Guo JW, Deng ZJ, Fu YH, Yang M, Ren B, Pan JQ, Liu RX (2009) Effects of panax notoginsenoside on TNF- $\alpha$ and MMP-2 expressions in rats with postmyocardial infarction ventricular remodeling and the mechanism. J South Med Univ 29, 2048-50 [in Chinese].

20. Lim Y, Lee S, Kim JY, Shin JH, Kwon O (2016) A Phellinus baumii-based supplement containing Salvia miltiorrhiza Bunge improves atherothrombotic profiles through endothelial nitric oxide synthase and cyclooxygenase pathways in vitro and in vivo. $J$ Funct Foods 24, 231-43.

21. Wang J, Wu SD, Zuo YF, Chen SC, Huang YC, Zheng K (2004) Effect of compound Salvia miltiorrhiza Bunge injection on the vascular endothelium-dependent relaxation and the mRNA expression of ET-1 and eNOS in the patients of coronary heart disease. Chin $J$ Arterioscler 12, 585-8 [in Chinese].

22. Tzeng TF, Lu HJ, Liou SS, Chang CJ,Liu IM (2013) Cassia tora (Leguminosae) seed extract alleviates high-fat diet-induced nonalcoholic fatty liver. Food Chem Toxicol 51, 194-201.

23. Dong P, Pan L, Zhang X, Zhang W, Wang X, Jiang M, Chen Y, et al (2017) Hawthorn (Crataegus pinnatifida Bunge) leave flavonoids attenuate atherosclerosis development in apoE knock-out mice. $J$ Ethnopharmacol 198, 479.

24. Zhang W, Zheng L, Zhang Z, Hai CX (2012) Protective effect of a water-soluble polysaccharide from Salvia miltiorrhiza Bunge on insulin resistance in rats. Carbohydr Polymer 89, 890-8.

25. Lam FFY, Yeung JHK, Chan KM, Or PMY (2007) Relaxant effects of danshen aqueous extract and its constituent danshensu on rat coronary artery are mediated by inhibition of calcium channels. Vasc Pharmacol 46, 271-7. 